\title{
Robotic motion planning in unknown dynamic environments: existing approaches and challenges
}

\begin{abstract}
Path planning with obstacles avoidance in dynamic environments is a crucial issue in robotics. Numerous approaches have been suggested for the navigation of mobile robots with moving obstacles. In this paper, about 50 articles have been reviewed and briefly described to offer an outline of the research progress in motion planning of mobile robot approaches in dynamic environments for the last five years. The benefits and drawbacks of each article are also explained. These papers are classified based on their issues into ten groups which are: stability, efficiency, smooth path, run time, path length, accuracy, safety, future prediction (uncertainties), control, and less computation cost. Finally, some scope and challenging topics are presented based on the papers mentioned.
\end{abstract}

Keyword: Robot navigation; Dynamic motion planning; Obstacle avoidance 